\title{
ASYMPTOTIC SOLUTIONS FOR DOUBLE PENDANT AND EXTENDED SESSILE DROPS
}

\author{
BY
}

\author{
S. B. G. M. O'BRIEN*
}

Philips Research Labs, Eindhoven, Netherlands

\begin{abstract}
Asymptotic solutions are obtained for small double pendant drops which are shown to consist of two (or more) nearly spherical drops joined together by a thin neck region, the upper drop being slightly larger. Equivalent solutions for so-called extended sessile drops represent the curling of the drop profile in on itself beyond the point where the inclination of the drop becomes greater than $\pi$ radians.
\end{abstract}

1. Introduction. The shape of liquid drops is described by the Laplace capillary equation yielding a balance between gravity, hydrostatic pressure, and surface tension effects. In the axisymmetric case the problem reduces to an ordinary nonlinear second-order differential equation or, equivalently, two coupled first-order equations, see Eq. (1), with suitable boundary conditions. Progress by analytic means is only possible by means of asymptotic simplification: we restrict attention to the case of small sessile and pendant drops. (A sessile drop is defined as one resting on a substrate while a pendant drop hangs from a ceiling.) In a recent paper [1], it was shown that in order to make progress a number of different scaled regions must be introduced and matched. These results are summarised in Sec. 2 and are a clear improvement on previous studies [2]. Our aim here is to show how the pendant solutions may be extended to describe double (and indeed multiple) drop solutions. In an analogous way, sessile drop solutions can be extended past the point where the drop profile becomes horizontal in order to describe physical profiles as observed by Padday [3].

2. Summary of previous results. As in [1], the equations for a pendant drop can be written as

$$
\frac{d X}{d \phi}=\frac{X \cos \phi}{-X Y+X P-\sin \phi} ; \quad \frac{d Y}{d \phi}=\frac{X \sin \phi}{-X Y+X P-\sin \phi}
$$

where $X, Y$ are the radial coordinate and height made dimensionless with the capillary length $\left(a=(\gamma / \rho g)^{1 / 2}\right), \gamma$ being surface tension, $\rho$ the liquid density, and $g$ gravitational acceleration. The pressure jump $P$ has been made dimensionless

Received November 1, 1991.

1991 Mathematics Subject Classification. Primary 76B45, 34E10, 34E15.

${ }^{*}$ Current address: Dept. of Mathematics, University of Limerick, Ireland.

(C)1994 Brown University 
- outer 1

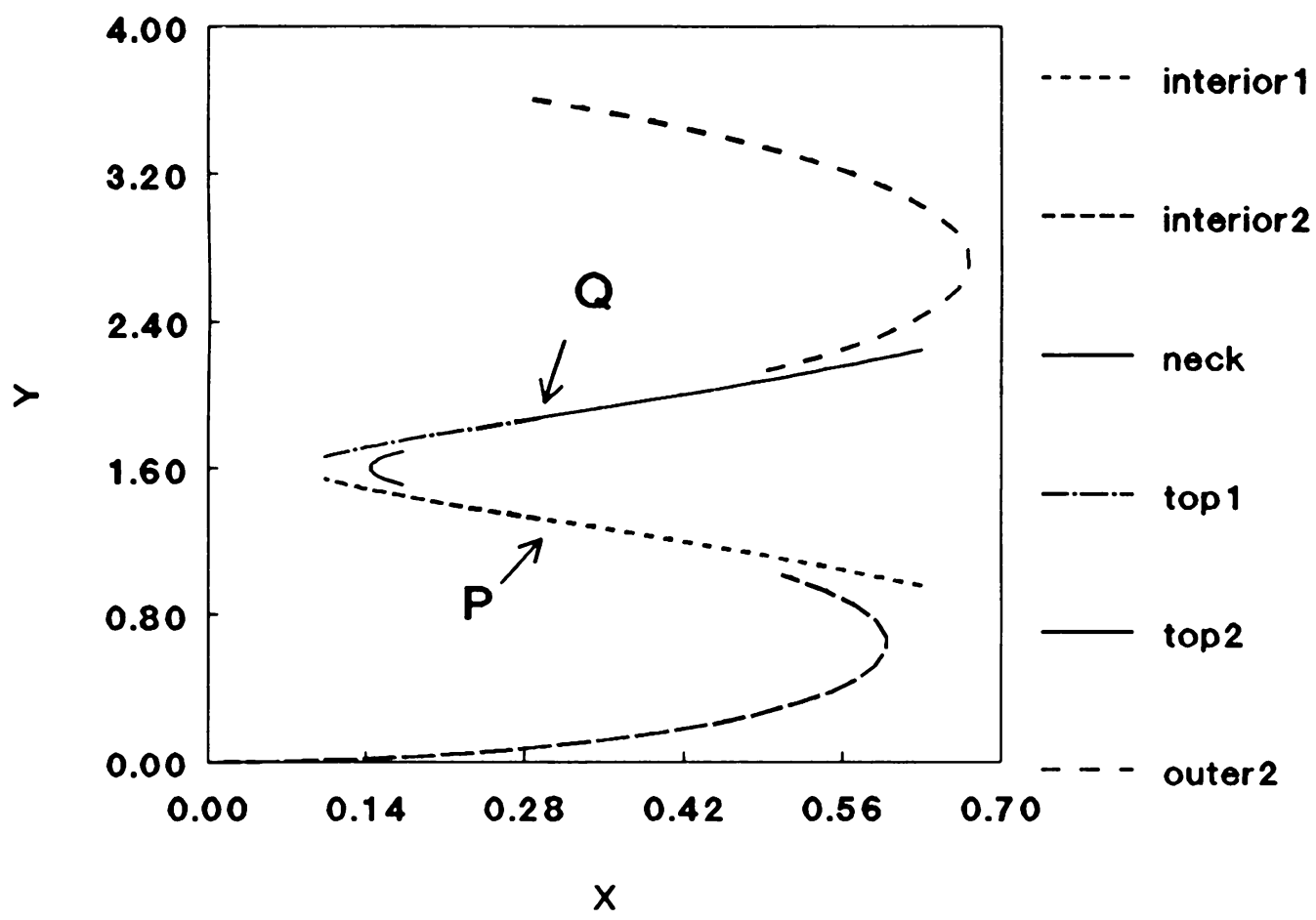

Fig. 1. Plot of double pendant drop for the case $R=0.6$ using constituent asymptotic solutions. Note occurrence of points of inflection $P, Q$.

with $\rho g a$ while $\phi$ is the angle of inclination at any point. Referring to Fig. 1, the boundary conditions are: $X=Y=0$ when $\phi=0 ; X=R$ when $\phi=\pi / 2$, where $R$ is the dimensionless radius of the drop at the point of maximum bulge and the small parameter about which our analysis is based. The extra boundary condition is used to solve for the unknown $P$. Referring again to Fig. 1, the following regions, rescaled variables and solutions in powers of $R$ were identified in [1]:

(i) Outer 1 region: $x=X / R ; y=Y / R ; p=R P$;

$$
\begin{gathered}
x=\sin \phi-\frac{1}{3} R^{2} \cos ^{2} \phi \tan \phi / 2 ; \\
y=(1-\cos \phi)-R^{2}\left(\frac{1}{3} \cos \phi-\frac{1}{3} \cos ^{2} \phi+\frac{2}{3} \ln (\cos \phi / 2)\right) ; \\
p=2+\frac{1}{3} R^{2} .
\end{gathered}
$$

(ii) Interior boundary layer: $\xi=X / R^{2} ; \zeta=-2 / R^{2}+Y / R^{3} ; p=R P ; \Phi=$ $(\pi-\phi) / R$;

$$
\begin{gathered}
\xi=\frac{1}{2}\left[\Phi \pm\left(\Phi^{2}-\frac{8}{3}\right)^{1 / 2}\right] ; \\
\zeta=\mp \frac{1}{4} \Phi\left(\Phi^{2}-\frac{8}{3}\right)^{1 / 2}-\frac{2}{3} \ln \left[\Phi+\left(\Phi^{2}-\frac{8}{3}\right)^{1 / 2}\right]-\frac{1}{4} \Phi^{2}+\frac{1}{3}+\frac{4}{3} \ln 2-\frac{2}{3} \ln R .
\end{gathered}
$$


The first root in each case refers to the solution before the point of inflection $\phi=\pi-(8 / 3)^{1 / 2} R$ and the second to that after this point.

(iii) Neck solutions: $u=X / R^{3} ; v=Y / R^{3}-2 / R^{2}$;

$$
u=\frac{2}{3} \operatorname{cosec} \phi ; v=-\frac{2}{3} \ln [\tan \phi / 2]+\frac{2}{3} \ln 6 .
$$

(iv) Top boundary layer: $\xi=X / R^{2} ; \zeta=-2 / R^{2}+Y / R^{3} ; p=R P ; \Phi=\phi / R$;

$$
\begin{gathered}
\xi=\frac{1}{2}\left[\Phi-\left(\Phi^{2}-\frac{8}{3}\right)^{1 / 2}\right] ; \\
\zeta=-\frac{1}{4} \Phi\left(\Phi^{2}-\frac{8}{3}\right)^{1 / 2}-\frac{2}{3} \ln \left[\Phi+\left(\Phi^{2}-\frac{8}{3}\right)^{1 / 2}\right]+\frac{1}{4} \Phi^{2}-\frac{1}{3}+\frac{2}{3} \ln 24-2 \ln R .
\end{gathered}
$$

3. Double drop profiles. The above solutions are valid up to the point where $\phi=(8 / 3)^{1 / 2} R$ but as pointed out by Prof. D. H. Peregrine, the top layer solutions are mirror images of the interior layer solutions and thus can be extended by interpreting this value of $\phi$ as another point of inflection. The scales in (5) remain valid and on the upper side of the second point of inflection we obtain

$$
\begin{gathered}
\xi=\frac{1}{2}\left[\Phi+\left(\Phi^{2}-\frac{8}{3}\right)^{1 / 2}\right], \\
\zeta=\frac{1}{4} \Phi\left(\Phi^{2}-\frac{8}{3}\right)^{1 / 2}-\frac{2}{3} \ln \left[\Phi+\left(\Phi^{2}-\frac{8}{3}\right)^{1 / 2}\right]+\frac{1}{4} \Phi^{2}-\frac{1}{3}+\frac{2}{3} \ln 24-2 \ln R .
\end{gathered}
$$

As the profile begins to close itself off again beyond the point of inflection, it is not unreasonable to expect another nearly circular solution along the lines of the outer region (2). Inspection suggests the following scales:

$$
\sigma=X / R ; \quad \tau=Y / R-2 ; \quad P=p / R .
$$

The equations in the newly scaled variables are

$$
\frac{d \sigma}{d \phi}=\frac{\sigma \cos \phi}{-R^{2}(2 \sigma+\sigma \tau)+\sigma p-\sin \phi} ; \quad \frac{d \tau}{d \phi}=\frac{\sigma \sin \phi}{-R^{2}(2 \sigma+\sigma \tau)+\sigma p-\sin \phi} .
$$

The following solutions are obtained:

$$
\begin{gathered}
\sigma=\sin \phi+R^{2}\left(\frac{4}{3} \sin \phi+\frac{1}{3} \cos ^{3} \phi / \sin \phi+E / \sin \phi\right), \\
\tau=1-\cos \phi+R^{2}\left(-\frac{4}{3} \cos \phi+\frac{1}{3} \cos ^{2} \phi+\left(-\frac{1}{3}-E\right) \ln [\sin \phi / 2]\right. \\
\left.+\left(E-\frac{1}{3}\right) \ln [\cos (\phi / 2)]+F\right) .
\end{gathered}
$$

It is noteworthy that the lowest-order terms of these solutions are identical to the equivalent terms in (2), but thereafter the solutions differ somewhat. Matching of the solutions (6) and (9) using an intermediate variable $\theta$ and gauge function $\eta(R)$ where $1 \gg \eta \gg R$ yields the following:

$$
E=-1, \quad F=-\frac{8}{3} \ln R+\frac{4}{3} \ln 6+\frac{1}{3}
$$

where switchback terms have been added. The solution is thus fully determined. We note also that for the purposes of calculating composite expansions, the common 
parts of (6) and (9) are given by

$$
\begin{aligned}
& X(\text { common part })=R \phi-2 R^{3} /(3 \phi), \\
& Y(\text { common part })=2 R+2 / 3 R^{3} \ln \phi-2 / 3 R^{3} \ln 2+(F-1) R^{3}+R / 2 \phi^{2} .
\end{aligned}
$$

In the upper drop it is then easy to show that the maximum radius (at the point where $\phi=\pi / 2$ ) is given by $R+\frac{1}{3} R^{3}$. Since $R$ is the dimensionless radius of the lower drop, the upper drop is slightly larger. This is physically reasonable since the hydrostatic pressure will be smaller in the upper drop and this must be balanced by a decrease in curvature, i.e., a larger radius. Referring to Fig. 1 we note that at the second point of inflection $\phi=(8 / 3)^{1 / 2} R$ and is increasing. Clearly as $\phi$ approaches $\pi$, solutions (9) will blow up and it is necessary to insert another boundary layer. But this is completely analogous to the situation in the lower drop summarised in Sec. 2 . Thus we may conclude that the second drop will also have a neck region connecting it to yet another slightly larger drop and this process continues indefinitely. Fig. 1 is a plot of the different constituent solutions for a double drop.

One further comment is perhaps in order here: there is a well-known phenomenon which can be verified by examination of the relatively slow flow of water from a circular tap. (This gives rise to a cylindrical falling jet which eventually, if allowed, breaks into distinct droplets.) However, near the tap exit point, a regular cylindrical jet is observed. If an object is placed in the water stream within, say, ten centimeters of the exit point, the free surface of the jet takes up a periodically corrugated appearance. A possible explanation for this phenomenon is that placing an obstacle in the stream brings hydrostatic pressure into the problem whereby Eq. (1) becomes very roughly valid for slow-enough flow. Thus the corrugated appearance can be interpreted as a multiple pendant drop along the lines of the present paper.

4. Extended sessile profiles. In [1] solutions for sessile drops were given for the case where $\phi$ increases from 0 to $\pi$, see Fig. 2, the latter value representing the situation in the case where the contact angle of drop and substrate is $\pi$. However, in [3] it is illustrated how physical situations can arise whereby $\phi$ can be greater than $\pi$ (though the contact angle remains $\leq \pi$ ). The physical situation is represented in Fig. 2 and the question arises as to whether the asymptotic solutions of [11] can be extended in an analogous fashion to the pendant drop. In [1] it was shown that a boundary layer also occurs at the base of the sessile drop when $\phi$ approaches $\pi$. The governing equation is Eq. (1) with a change in the sign of the $X Y$ term and the

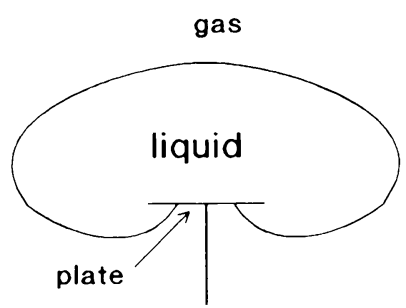

FIG. 2. Schematic of sessile drop for the case when $\phi>\pi$ as a result of the strategically placed circular plate. 
following solutions were obtained:

(i) Outer 1 region: $x=X / R ; y=Y / R ; p=R P$;

$$
\begin{gathered}
x=\sin \phi+\frac{1}{3} R^{2} \cos ^{2} \phi \tan \phi / 2 ; \\
y=(1-\cos \phi)+R^{2}\left(\frac{1}{3} \cos \phi-\frac{1}{3} \cos ^{2} \phi+\frac{2}{3} \ln (\cos \phi / 2) ;\right. \\
p=2-\frac{1}{3} R^{2} .
\end{gathered}
$$

(ii) Boundary layer: $\xi=X / R^{2} ; \zeta=-2 / R^{2}+Y / R^{3} ; p=R P ; \Phi=(\pi-\Phi) / R$;

$$
\begin{gathered}
\xi=\frac{1}{2}\left[\Phi+\left(\Phi^{2}+\frac{8}{3}\right)^{1 / 2}\right] ; \\
\zeta=-\frac{1}{4} \Phi\left(\Phi^{2}+\frac{8}{3}\right)^{1 / 2}+\frac{2}{3} \ln \left[\Phi+\left(\Phi^{2}+\frac{8}{3}\right)^{1 / 2}\right]-\frac{1}{4} \Phi^{2}-\frac{1}{3}-\frac{4}{3} \ln 2+\frac{2}{3} \ln R .
\end{gathered}
$$

Unlike the case of the pendant drop, $\phi$ is not restricted since the sign inside the square root is positive in this case. We can in fact continue the solutions simply by allowing $\phi$ to become larger than $\pi$. Equations (13) thus remain valid for $\phi>\pi$ and the drop starts to curl in on itself (see Fig. 2). The boundary layer solutions are, however, only valid in an $O(R)$ neighborhood of $\pi$. Analogous to the pendant drop, we seek a "neck" solution by scaling as follows:

$$
u=X / R^{3} ; \quad v=Y / R^{3}-2 / R^{2} .
$$

Note that the motivation for this scaling is that the curvature terms should dominate at lowest order. We actually scaled more generally in (14): $u=X / \delta(R) ; v=$ $Y / \delta(R)-2 R / \delta(R)$ where $\delta(R) \ll R$ but is unknown. Matching then gives the precise value of $\delta(R)$ as $R^{3}$. These scales yield the following equations:

$$
\frac{d u}{d \phi}=-u \cot \phi ; \quad \frac{d v}{d \phi}=-u .
$$

The solutions at lowest order are

$$
u=A \operatorname{cosec} \phi ; \quad v=-A \ln |\tan \phi / 2| B .
$$

If we match these solutions with (13), again using an intermediate variable defined such that $\phi=\pi+\theta \eta(R)$ with $R \ll \eta \ll 1$, the following values for the integration constants $A$ and $B$ are found:

$$
A=-\frac{2}{3} ; \quad B=\frac{2}{3}(2 \ln R-\ln 6) .
$$

For the purposes of getting composite expansions, the common parts of (13) and (16) to the present order are found to be

$$
\begin{aligned}
& X(\text { common part })=2 R^{3} /\{3(\pi-\phi)\}, \\
& Y(\text { common part })=2 R+\frac{2}{3} R^{3} \ln 2-\frac{2}{3} R^{3} \ln (\pi-\phi)+B .
\end{aligned}
$$

An examination of (16) indicates that as $\phi$ increases towards $2 \pi$, the solutions again blow up and another boundary layer must be inserted incorporating a new stretched independent variable. Beyond this boundary layer region, the physics suggests another neck type solution rather than an outer nearly spherical solution. We will not proceed 


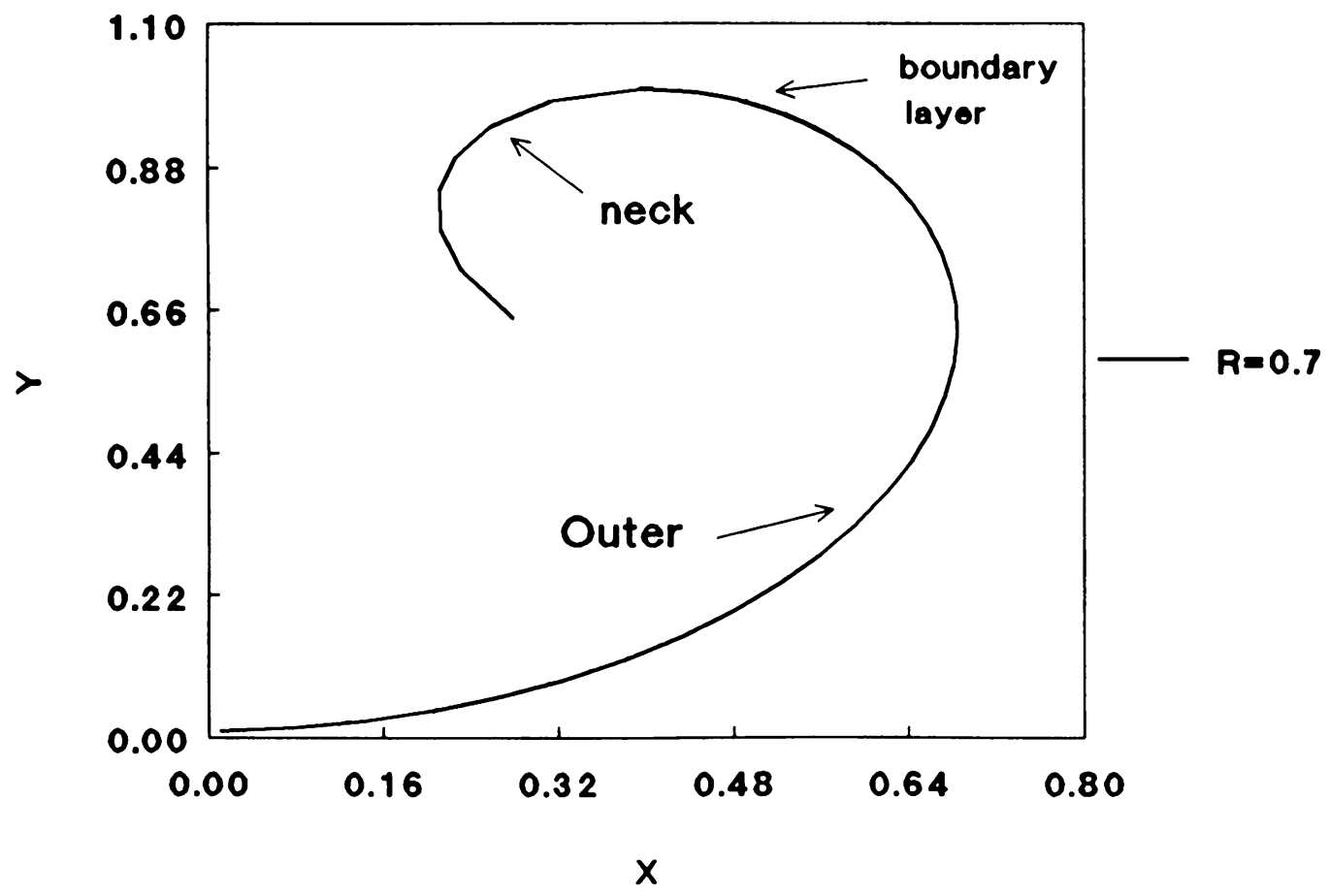

Fig. 3. Plot of extended sessile drop (upside down) for the case $R=0.7$.

beyond this point but it seems likely that the analysis is no more difficult than that already presented here. Fig. 3 shows an example of a plot of the sessile drop solutions.

5. Conclusions. Matched asymptotic expansion techniques were used to obtain solutions for small double pendant and extended sessile drops. It is necessary to identify the existence of several regions where different scales are needed. At a certain point the form of the solutions begins to resemble those previously obtained, but it is not identical. With a little care, however, continuing the drop profiles is relatively straightforward.

\section{REFERENCES}

[1] S. B. G. M. O'Brien, On the shape of small sessile and pendant drops by singular perturbation techniques, J. Fluid Mech. 233, 519 (1991)

[2] A. K. Chesters, An analytical solution for the profile and volume of a small drop or bubble symmetrical about a vertical axis, J. Fluid Mech. 81, 609 (1977)

[3] J. F. Padday, The profiles of axially symmetric menisci, Phil. Trans. Roy. Soc. London A269, 265 (1971) 